\title{
Editorial
}

\section{Methodologic Issues of Case-Control Studies: A Review of Established and Newly Recognized Limitations}

\author{
Erika M. C. D’Agata, MD, MPH
}

The case-control study design is used extensively to identify risk factors associated with patients' harboring antimicrobial-resistant pathogens. Although case-control studies have numerous strengths, this editorial reviews and discusses their current limitations. Three articles in this issue of Infection Control and Hospital Epidemiology, which provide further insight and potential solutions to the limitations of this study design, are also reviewed. ${ }^{1-3}$

The main strength of a case-control study design is the analysis of risk factors associated with outcomes that occur infrequently. Case-control studies, in contrast to cohort studies, allow the rapid and efficient investigation of a hypothesis because the outcome of interest has already occurred. This design is therefore frequently used for the study of risk factors associated with antimicrobial-resistant pathogens, an infrequent outcome that is predominantly identified by clinical or surveillance cultures.

The main weakness of case-control studies is the potential for introducing biases, including measurement bias and selection bias. Measurement bias arises due to the retrospective nature of collecting data about predictor variables. For example, patients who harbor antimicrobial-resistant pathogens may be more likely to recall exposure to antibiotics, compared with patients who do not harbor these pathogens. This differential recall bias would lead to a greater association of antibiotic exposure with the outcome of interest. Selection bias can occur during the identification of case-patients and control-patients if their inclusion depends on the exposure of interest. Methods to avoid this type of bias have been discussed elsewhere. ${ }^{4}$

There are several other methodologic issues that warrant discussion, pertaining specifically to the identifica- tion of risk factors for antimicrobial-resistant pathogens when the case-control study design is used.

\section{OUTCOME OF INTEREST: DETECTION OF COLONIZATION OR POSITIVE CLINICAL CULTURES?}

Most case-control studies addressing risk factors associated with patients' harboring antimicrobial-resistant pathogens use clinical cultures to identify cases. However, doing so may lead to erroneous associations between predictor variables and the outcome. For example, studies have identified central venous catheters as risk factors for bacteremia caused by antimicrobial-resistant pathogens. One could argue that these invasive devices are risk factors for bacteremia, regardless of the presence or absence of antimicrobial resistance. Because colonization precedes infection, a more accurate method of identifying cases would be to assess the colonization status of patients. Colonization would therefore identify risk factors directly related to the presence of antimicrobial-resistant pathogens, and not those related to developing an infection. Unfortunately, surveillance cultures may not always be feasible because they require substantial resources and time. Future studies should quantify and describe the differences in study findings depending on whether cases are identified by colonization status or positive clinical cultures.

\section{MULTIDRUG RESISTANCE VERSUS SINGLE DRUG RESISTANCE}

To date, most case-control studies have assumed single drug resistance in the antimicrobial-resistant pathogen under study, even though multidrug resistance 
may be present. Because exposure to one antimicrobial may select for resistance to other, unrelated antimicrobials through co-resistant or cross-resistant mechanisms among multidrug-resistant bacteria, assuming single drug resistance may lead to discrepant conclusions between studies about the risk associated with specific antibiotics. To demonstrate the importance of addressing co-resistance in case-control studies, we analyzed antibiotic exposure as a risk factor for patients' harboring Pseudomonas aeruginosa resistant only to ciprofloxacin (CR-PA) and patients' harboring multidrug-resistant $P$. aeruginosa (MDR-PA), defined as resistant to ciprofloxacin and other antibiotics. ${ }^{5}$ These two groups of cases were compared with patients not harboring $P$. aeruginosa. By conditional logistic regression, significant differences in antibiotic exposures were identified between the CR-PA and the MDR-PA cases. Among several antibiotics analyzed, only quinolone exposure was significantly associated with CR-PA, whereas several other antimicrobials including third- and fourth-generation cephalosporins and aminoglycosides were associated with MDR-PA. ${ }^{5}$ Not addressing the full susceptibility profile of the antimicrobial-resistant pathogen under investigation may lead to discrepancies in study findings pertaining to which antibiotics are risk factors. Investigators should consider providing the antibiogram patterns of the antimicrobial-resistant pathogen under investigation. Alternatively, the analysis could be restricted to specific co-resistant patterns.

\section{DEFINING ANTIBIOTIC EXPOSURE}

The definition of antimicrobial exposure prior to the recovery of antimicrobial-resistant pathogens varies substantially between studies. This variable is sometimes dichotomized to whether or not the patient received a particular antibiotic. In other studies, the total number of days of antibiotic exposure is used as a continuous variable. The period of time during which antibiotic exposure is assessed, prior to the recovery of antimicrobial-resistant pathogens, also varies considerably. In some studies, antibiotic exposure is assessed during the 14 days prior to the recovery of antimicrobial-resistant pathogens; in other studies, the period of exposure is 90 days or more. Although the risk associated with antibiotic exposure is likely cumulative, the minimum duration of exposure leading to an increased risk of harboring antimicrobial-resistant pathogens is not known. The administration of multiple antimicrobials either concurrently or sequentially adds further difficulties in defining antibiotic exposure and interpreting the effect of individual antibiotics. In addition to animal studies, prospective human studies addressing antibiotic exposure among patients observed with serial surveillance cultures until antimicrobial-resistant pathogens are detected are needed to better define and quantify the risk associated with antibiotic exposure and the time to antimicrobial-resistant pathogen emergence. For now, it is important to clearly describe and justify the criteria used to define antibiotic exposure. Using precise definitions will allow a reasonable, although not optimal, comparison between study findings.

\section{METHOD OF ACQUISITION}

Antimicrobial-resistant pathogens can be acquired either endogenously or exogenously. Endogenous acquisition occurs when previously susceptible bacteria develop resistance. Exogenous acquisition occurs through patientto-patient transmission. Antibiotic exposure has an important role in both endogenous acquisition and exogenous acquisition. In the latter, antibiotics may promote the colonization and overgrowth of antimicrobial-resistant bacteria by eradicating the normal flora. ${ }^{6}$

An important risk factor for exogenous acquisition is colonization pressure-the proportion of other patients colonized with the antimicrobial-resistant pathogen under investigation. Several studies have documented that the greater the proportion of other patients harboring antimicrobial-resistant pathogens, the greater the likelihood of acquiring the bacteria under investigation. ${ }^{7,8}$ Colonization pressure should therefore be included as a variable, if possible, when assessing risk factors for antimicrobial-resistant pathogen acquisition or during outbreak investigations.

\section{CHOOSING THE MOST APPROPRIATE CONTROL GROUP}

There are two types of control groups frequently used in case-control studies evaluating risk factors for antimicrobial-resistant pathogens. Each has its own limitations and potential biases. The first is a control group chosen from patients who do not harbor the antimicrobialresistant pathogen under investigation and the second is a control group chosen from patients who harbor the susceptible pathogen. The first type of control group-patients who do not harbor antimicrobial-resistant pathogens-may lead to identification of risk factors for the bacteria under investigation, regardless of whether they are resistant. The second type of control group-patients harboring susceptible pathogens-may lead to an overestimation of the association between antimicrobial exposure and cases: as prior active antibiotic exposure would eradicate susceptible organisms, the remaining potential control-patients would be those patients who have not received certain antimicrobials. ${ }^{9}$ For example, if the control group chosen to identify risk factors for colonization with quinolone-resistant Escherichia coli is represented by patients colonized with quinolone-susceptible $E$. coli, then these control-patients are unlikely to have been exposed to quinolones, as exposure to this antimicrobial may have eradicated colonization with the susceptible bacteria. Thus, the association between quinolone exposure and quinolone-resistant $E$. coli would be overestimated. Harris et al. have clearly validated this theory for several different antimicrobial-resistant pathogens. ${ }^{9}$

In this issue of Infection Control and Hospital Epidemiology, Kaye et al. provide a potential solution to the difficulties in choosing the appropriate control group: a case-case-control study design. ${ }^{2}$ In this type of study design, essentially two case-control studies are performed: the first identifies cases as patients harboring antimicro- 
bial-resistant pathogens and the second identifies cases as patients harboring the susceptible bacteria. These two groups of cases are compared with the same control group-patients who do not harbor the pathogen under investigation. The independent risk factors identified from these two studies can then be compared to determine the contribution of the antimicrobial resistance component to the recovery of the antimicrobial-resistant pathogen under investigation. The major limitation with this study design, as the authors discuss, is the difficulties in matching for potential confounders because only one control group is used. If matching is crucial to the validity of the study, then the authors recommend using two controls groups, thereby performing two separate case-control studies.

Another study in this issue of Infection Control and Hospital Epidemiology, by Harris et al., discusses the biases that may arise in the selection of control-patients depending on whether they are identified by negative clinical cultures for the antimicrobial-resistant pathogen under investigation or by the absence of clinical cultures. ${ }^{1}$ These authors discuss that if the control group is chosen from patients for whom clinical cultures were not performed, misclassification of control-patients could occur because some control-patients may, in fact, be undetected case-patients (patients harboring antimicrobial-resistant pathogens that were not detected due to the absence of clinical cultures). However, this misclassification bias may not be too severe because the clinical signs and symptoms of patients with an antimicrobial-resistant pathogen infection should, in most circumstances, mandate clinical cultures, thereby minimizing the number of unrecognized cases. There is another potential bias that may be introduced when identifying control-patients by the absence of clinical cultures that warrants discussion. Differences between case-patients and control-patients may reflect the fact that clinical cultures were performed for case-patients but not for control-patients. Therefore, the severity of illness or antibiotic exposure may be higher among casepatients, regardless of the presence or absence of antimicrobial-resistant pathogens.

Harris et al. also discuss the limitations of the control group chosen among patients for whom clinical cultures were performed but antimicrobial-resistant pathogens were not recovered. ${ }^{1}$ Similar to the above rationale, they demonstrate that using clinical cultures to identify controlpatients will lead to the selection of a control group that is sicker, with a higher comorbidity score and greater exposure to antibiotics, compared with a control group for which clinical cultures were not performed.

Therefore, what is the most appropriate control group when performing a study to identify risk factors associated with harboring antimicrobial-resistant pathogens? The answer depends not only on the question being asked, as Harris et al. ${ }^{1}$ state, but also on a careful interpretation of the generalizability of the study findings. Thus, choosing a control group among all patients for whom clinical cultures were performed will result in a valid study, but a study in which the findings can be generalized only to patients for whom clinical cultures were performed. The findings of such a study will need to be interpreted accordingly, with an understanding that differences between case-patients and control-patients regarding severity of illness and antibiotic exposure may be minimized. Hennekens and Buring state that "the controls must be selected to represent not the entire nondiseased population but the population of individuals who would have been identified and included as cases had they also developed the disease" and that "the crucial requirement [for controls] is that they be comparable to the source population of cases and that any exclusions or restrictions made in the identification of cases apply equally to the controls and vice versa." Although using a control group of patients for whom clinical cultures were performed may only be generalizable to this subset of hospitalized patients, this choice of control group would ensure that the control-patients were comparable to the source population of case-patients (patients for whom clinical cultures were performed) and that differences between case-patients and control-patients did not reflect potential factors associated with performing clinical cultures, regardless of the presence or absence of antimicrobial-resistant pathogens. Assessing the colonization status of case-patients and control-patients would avoid the limitations associated with clinical cultures, as discussed above.

\section{ASSESSING OUTCOMES}

Case-control studies are also used to assess the outcome of infections caused by antimicrobial-resistant pathogens. Because patients who develop an infection caused by an antimicrobial-resistant pathogen may have a greater severity of illness and more comorbidities, it is important to control for these potential confounders when assessing adverse outcomes related to infection. Length of hospital stay (LOS) is also an important confounder. Because LOS is likely to be longer for patients who develop an infection and longer LOS can lead to poorer outcomes, this variable requires consideration in the analysis. In this issue of Infection Control and Hospital Epidemiology, Blot et al. evaluated the impact of matching for LOS on mortality among intensive care unit patients who developed $E$. coli bacteremia. ${ }^{3}$ In their study, matching for LOS did not affect the outcome: mortality rates were similar in the matched and unmatched case-control studies. However, the absence of differences in mortality between these two groups may reflect factors other than LOS. As the authors comment and have shown in a previous publication, the mortality rates among patients with $E$. coli bacteremia did not differ from the mortality rates among patients without bacteremia when timely administration of antimicrobial therapy occurred..$^{10}$ This may explain why controlling for LOS did not affect mortality rates.

Another important issue to consider when performing outcomes studies is to control for comorbidities, in addition to the severity of illness. ${ }^{11}$ Although in the study by Blot et al. case-patients and control-patients were appropriately adjusted for differences in severity of illness, using the Acute Physiology and Chronic Health Evaluation II 
score, comorbidities were not addressed. If the control group patients had more comorbidities, one could argue that their mortality rate may be higher, thereby minimizing differences in poor outcomes between case-patients and control-patients. Finally, when outcomes are being assessed, it is important to examine factors occurring after the infection that may affect mortality regardless of the infection under investigation. For example, if the duration of follow-up is substantial, then one needs to consider other subsequent adverse events that may affect mortality but that are not directly related to the antimicrobial-resistant pathogen infection. For example, if the control group develops more nosocomial infections after the antimicrobialresistant pathogen infections among case-patients, one could argue that these infections adversely affected the mortality rate among control-patients, regardless of LOS prior to infection.

Adjusting for LOS prior to infection should still be a necessary step in case-control studies. The article by Blot et al. raises the possibility that, in studies in which the LOS prior to infection is short, controlling for LOS may not be necessary. In their study, the LOS was a median of 6 days and may have been too brief to affect the subsequent mortality rates of patients.

\section{NUMBER OF RISK FACTORS ANALYZED}

Certain case-control studies attempt to generate hypotheses and, in so doing, analyze a multitude of variables. Analyzing numerous variables may lead to a statistically significant association due to chance alone. For example, in a study analyzing 20 variables as potential risk factors for an outcome, which uses a $P$ value of less than .05 for statistical significance, $5 \%$, or at least one of 20 variables, can reach statistical significance by chance alone. Studies demonstrating an association between a particular variable and an outcome where there is no biological plausibility for the association, or which lack an a priori hypothesis, need to be interpreted with caution.

\section{CONCLUSION}

A critical evaluation of case-control studies involves assessing the roles of chance, bias, and confounding in explaining study findings. In case-control studies specifically addressing risk factors for harboring antimicrobialresistant pathogens, additional issues, as outlined in this editorial, need to be considered. Although in this issue of Infection Control and Hospital Epidemiology Harris et al. and Kaye et al. provide some potential solutions to some of the limitations encountered with case-control studies, substantial research is still required to further refine this type of study design.

\section{REFERENCES}

1. Harris AD, Carmeli Y, Samore MH, Kaye KS, Perencevich E. Impact of severity of illness bias and control group misclassification bias in case-control studies of antimicrobial-resistant organisms. Infect Control Hosp Epidemiol 2005;26:342-345.

2. Kaye KS, Harris AD, Samore M, Carmeli Y. The case-case-control study design: addressing the limitations of risk factor studies for antimicrobial resistance. Infect Control Hosp Epidemiol 2005;26:346-351.

3. Blot S, De Bacquer D, Hoste E, et al. Influence of matching for exposure time on estimates of attributable mortality caused by nosocomial bacteremia in critically ill patients. Infect Control Hosp Epidemiol 2005; 26:352-356.

4. Hennekens CH, Buring JE. Epidemiology in Medicine. Boston: Little, Brown; 1987.

5. D'Agata EMC, Tacconelli E. The impact of multidrug-resistance in risk factor analyses of antibiotic exposure among antimicrobial-resistant bacteria. Presented at the 43th Interscience Conference of Antimicrobial Agents and Chemotherapy; September 14-17, 2003; Chicago, IL.

6. Donskey CJ. The role of the intestinal tract as a reservoir and source of transmission of nosocomial pathogens. Clin Infect Dis 2004;39:219-226.

7. D'Agata EMC, Thayer V, Schaffner W. An outbreak of Acinetobacter baumannii: the importance of cross-transmission. Infect Control Hosp Epidemiol 2000:21:588-592.

8. Bonten MJ, Slaughter S, Ambergen AW, et al. The role of "colonization pressure" in the spread of vancomycin-resistant enterococci: an important infection control variable. Arch Intern Med 1998:158:1127-1132.

9. Harris AD, Karchmer TB, Carmeli Y, Samore MH. Methodological principles of case-control studies that analyze risk factors for antibiotic resistance: a systematic review. Clin Infect Dis 2001;32:1055-1061.

10. Blot S, Vanderwoulde $\mathrm{K}$, Hoste E, et al. Absence of excess mortality in critically ill patients with nosocomial Escherichia coli bacteremia. Infect Control Hosp Epidemiol 2003;24:912-915.

11. Cosgrove $\mathrm{SE}$, Carmeli $\mathrm{Y}$. The impact of antimicrobial resistance on health and economic outcomes. Clin Infect Dis 2003;36:1432-1437. 